\title{
Ecología trófica de Athene cunicularia (Aves: Strigidae) en un sistema insular del norte de Chile: ¿posible respuesta funcional y numérica frente a Pelecanoides garnotii (Aves: Pelecanoididae)?
}

\section{Trophic ecology of Athene cunicularia (Aves: Strigidae) in an island system of northern Chile: ¿potential numerical and functional response related to Pelecanoides garnotii (Aves: Pelecanoididae)?}

\author{
Franco Cruz-Jofré $E^{1,2}$ \& Yerko A. VILINA ${ }^{1,3}$
}

${ }^{1}$ Facultad de Medicina Veterinaria, Universidad Santo Tomás. Ejército 146, Santiago, Chile. 2Laboratorio de Genética y Evolución, Departamento de Ciencias Ecológicas, Facultad de Ciencias, Universidad de Chile. Las Palmeras 3425, Ñuñoa, Santiago, Chile.

${ }^{3}$ CASEB, Centro de Estudios Avanzados en Ecología y Biodiversidad, Pontificia Universidad Católica de Chile. Alameda 340, Santiago, Chile.

Email: f.cruzjofre@gmail.com.

Email: yvilina@santotomas.cl

\section{RESUMEN}

Se analizó la dieta del pequén (Athene cunicularia) en cuatro islas del norte de Chile, mostrando que existe un fuerte predominio de artrópodos $(89,1 \%)$ en comparación con los vertebrados $(10,1 \%)$ respecto al número de presas consumidas. De estos taxa destacan los tenebriónidos $(42,2 \%)$ y curculiónidos $(31,2 \%)$ como los principales grupos consumidos, sin embargo, la presencia de mamíferos $(3,6 \%)$ y de aves $(6 \%)$ en la dieta es también relevante, ya que a pesar de representar menos del 10\% del número total de presas, el aporte energético de estos vertebrados constituyó cerca del $80 \%$ de la biomasa total ingerida. Se registró evidencias de depredación sobre el yunco (Pelecanoides garnotii); presa destacada con un $71 \%$ dentro del ítem aves y $40 \%$ del total de vertebrados consumidos, así también destaca el consumo de la llaca (Thylamys elegans) con en un $21 \%$ del total de vertebrados. Se encontraron tres individuos de piuchén o vampiro (Desmodus rotundus) especie no descrita con anterioridad en la dieta del pequén en el norte de Chile. Se discute respecto a las posibles respuestas funcional y numérica del pequén en isla Choros, asociadas a los registros de distintas densidades de yunco. Se analizó además, la variabilidad de la dieta por isla, el patrón de tamaño de las presas consumidas y la distribución espacial de nidos del pequén.

Palabras clave: desierto de Atacama, ecología trófica, islas, yunco, pequén, respuesta del depredador.

\begin{abstract}
The diet of Burrowing owl (Athene cunicularia) was analyzed in four islands in the North of Chile, showing that a strong predominance of arthropods (89.1\%) exists compared to vertebrates $(10.1 \%)$ regard to the number of consumed preys. Tenebrionids $(42.2 \%)$ and curculionids $(31.2 \%)$ stand out because they are the main consumed groups. However, the presence of mammals (3.6\%) and birds (6\%) in the diet is relevant too, because despite representing less than $10 \%$ of the total number of prey, the energy contribution of vertebrates constituted about $80 \%$ of the total biomass ingested. Evidence of predation on Peruvian diving petrel (Pelecanoides garnotii) was found, this prey stands out with $71 \%$ from the birds group, while regarding the total consumed vertebrates it reaches a $40 \%$. It is also worth mentioning the mouse-opossum (Thylamys elegans) consumption reaching $21 \%$ of the total vertebrates. Three individuals of Piuchen or vampire bat (Desmodus rotundus) were observed, this species have not been previously described in the Burrowing owl diet in the North of Chile. We discuss possibles functional and numerical responses of Burrowing owl in Choros Island, where different densities of Peruvian diving petrel were found. Besides, the diet variability per island, pattern size of preys consumed and spatial distribution of Burrowing owl nests were analyzed.
\end{abstract}

KeYwords: Atacama desert, Burrowing owl, islands, Peruvian diving petrel, predator response, trophic ecology. 


\section{INTRODUCCIÓN}

El pequén, Athene cunicularia (Molina 1782) es un depredador oportunista y generalista, que presenta actividad diurna con un marcado incremento de esta durante el crepúsculo (Yánez \& Jacksic, 1979; Núñez \& Yánez, 1982; Torres-Contreras et al. 1994), que utiliza principalmente la estrategia de captura de "sentarse y esperar" o "sit-andwait" (Silva et al. 1995).

La dieta del pequén ha sido estudiada en numerosos lugares de Chile, con diferencias en su composición, en general corresponde a una dieta amplia con una marcado consumo de invertebrados, entre un 75 y $95 \%$ del total de presas (Yánez \& Jaksic 1979, Schlatter et al. 1980, Schlatter et al. 1982, Núñez \& Yánez 1982, Jaksic 1986, Torres-Contreras et al. 1994, Silva et al. 1995, Zunino \& Jofré 1999, Muñoz -Pedrero \& Rau 2004, Rau et al. 2005, Carevic 2011), sin embargo en términos de biomasa, el aporte de los vertebrados a la dieta sería mayor (Silva et al. 1995). En zonas áridas, el porcentaje de invertebrados en la dieta representa entre un 87 y un $92 \%$, las proporciones de consumo cambian en el tiempo, incluyendo un mayor porcentaje de roedores en primavera-verano cuando estos son más abundantes (Yañez \& Jaksic 1979, Silva et al. 1995, Carevic et al. 2013). Los estudios de A. cunicularia en islas son realmente escasos, Zunino \& Jofré (1999), realizaron un estudio preliminar en isla Choros $\left(29^{\circ} 15^{\prime} \mathrm{S}\right)$, obteniendo resultados similares a las zonas áridas continentales, donde los Artrópodos representaron el $90 \%$ de las presas consumidas, destacándose el ratón de cola larga (Oligoryzomys longicaudatus) como el principal vertebrado consumido $(7,17 \%)$ además de la presencia de aves indeterminadas $(2,61 \%)$.

El presente estudio busca aumentar la información sobre la ecología trófica de esta especie en un sistema insular, complementándola con información referente a la comparación de dieta entre islas, patrón de tamaño de las presas, respuesta del depredador y nueva información sobre el ítem de presas consumidas, además hacemos una aproximación a la elección se sitios de nidificación y percha, en áreas de superficie restringida como lo es el caso de las islas.

\section{MATERIALES Y MÉTODOS}

\section{Área De Estudio}

La Reserva Nacional Pingüino de Humboldt (RNPH), es un área protegida cuya superficie total es de 859 hectáreas, involucra tres islas ubicadas en el desierto de Atacama, en el norte de Chile: Choros ( $\left.29^{\circ} 15^{\prime} \mathrm{S}, 71^{\circ} 33^{\prime} \mathrm{W}\right)$, Damas $\left(29^{\circ} 13^{\prime} \mathrm{S}, 71^{\circ} 32^{\prime} \mathrm{W}\right)$ y Chañaral $\left(29^{\circ} 01^{\prime} \mathrm{S}, 71^{\circ} 37^{\prime} \mathrm{W}\right)$. Existe una cuarta isla que no pertenece a la RNPH, la isla Gaviota $\left(29^{\circ} 15^{\prime} \mathrm{S}, 71^{\circ} 29^{\prime} \mathrm{W}\right)$ también incluida en el presente estudio. Las superficies y distancias al continente se presentan en la Tabla 1 y Figura 1A.

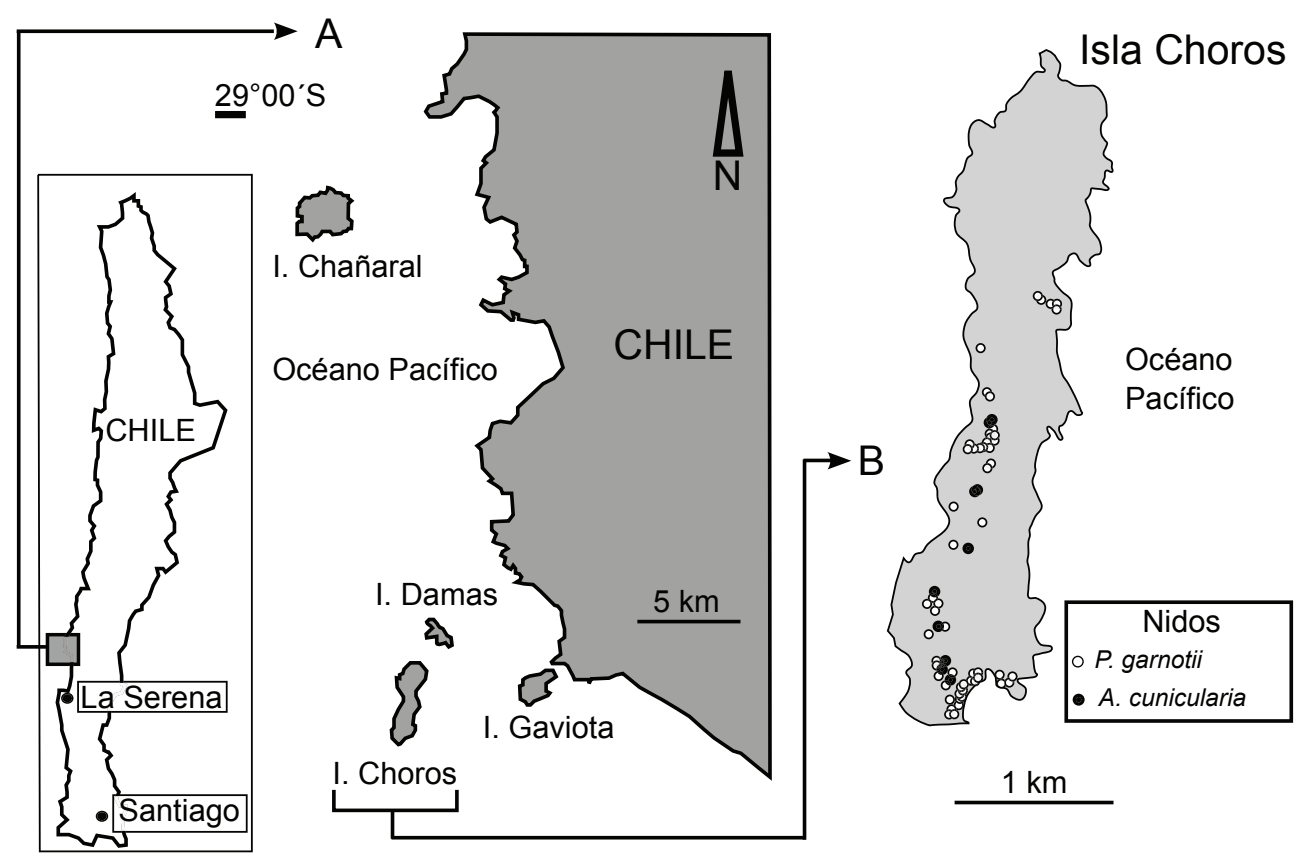

Figura 1: A: Área de Estudio B: Distribución espacial de nidos del pequén (Athene cunnicularia) y de la colonia de yunco (Pelecanoides garnotii) en isla Choros.

Figure 1: A: Study Area B: Spatial distribution of nests of Burrowing owl (Athene cunnicularia) and Peruvian diving petrel colony (Pelecanoides garnotii) on Choros Island. 
Dieta del Pequén en islas del norte de Chile: Franco Cruz-Jofré \& Yerko A. Vilina

TABLA 1. Superficie, distancia a la costa, número de muestras y nidos de pequén por isla.

TABLE 1. Area, distance from shore, samples size and Burrowing owl nests by island.

\begin{tabular}{|c|c|c|c|c|c|}
\hline IsLA & $\begin{array}{l}\text { SUPERFICIE } \\
\text { (ha) }\end{array}$ & $\begin{array}{c}\text { DisTANCIA AL CONTINENTE } \\
\qquad(\mathrm{km})\end{array}$ & $\mathrm{N}^{\mathrm{o}}$ de EGAGRÓPILAS & Restos Óseos & $\mathrm{N}^{\mathrm{o}}$ DE NIDOS \\
\hline Choros & 291 & 6 & 316 & $27^{*}$ & 15 \\
\hline Damas & 60 & 5 & 61 & - & 3 \\
\hline Gaviota & 212 & 0,4 & 15 & - & 1 \\
\hline Chañaral & 507 & 8 & 0 & $60 * *$ & 1 \\
\hline
\end{tabular}

El clima es templado con abundante nubosidad matinal. La precipitación anual es de $30 \mathrm{~mm}$. y la temperatura media anual es de $18^{\circ} \mathrm{C}$ (Capella et al. 1999). La vegetación presente es característica de zonas áridas costeras, identificada como formación vegetacional del desierto costero de Huasco (Gajardo 1994), que presenta un matorral con especies predominantes de los géneros Nolana, Oxalis y Heliotropium y cactáceas de los géneros Copiapoa, Eulychnia y Neoporteria. La fauna de vertebrados presente en las islas se caracteriza por la presencia de una lagartija (Liolaemus zapallarensis) que habita en las cuatro islas y la culebra de cola larga, Phylodrias chamissonis, registrada en isla Chañaral (Vilina et al. 1995). Destacan colonias de nidificación de pingüino de Humboldt, Spheniscus humboldti; y del yunco, Pelecanoides garnotii (Millie 1938, Goodall et al. 1956, Araya 1983), esta última especie nidifica en isla Choros (Vilina 1992; Collar et al. 1992; Simeone et al. 2003), en isla Chañaral también existió una gran colonia reproductiva de esta especie a inicios del siglo $\mathrm{XX}$, que desapareció por la introducción de zorros (Araya \& Duffy, 1987; Vilina 1992), no registrándose nidificación reciente. Tres especies de mamíferos han sido registradas, la llaca, Thylamys elegans, el conejo, Oryctolagus cuniculus, una especie originaria de Europa, la cual fue introducida por pescadores, ambas especies habitan en isla Chañaral, y es probable que se encuentren también en las otras islas (Vilina et al. 1995) y el ratón de cola larga (Olygoryzomys longicaudatus), identificado en isla Choros por medio de restos encontrados en egagrópilas (Zunino \& Jofré 1999). Recientemente Luna-Jorquera et al. (2012) establecieron la riqueza de especies de mamíferos y aves terrestres en las islas de la RNPH, indicando para isla Chañaral, 17 especies de aves terrestres y 3 mamíferos terrestres (T. elegans, $O$. cuniculus y D. rotundus), en isla Damas registraron 18 especies de aves terrestres y 1 roedor (Abrothrix olivaceus) y en isla Choros, 24 especies de aves terrestres y 2 especies de mamíferos introducidos (Mus musculus y O. cuniculus).

\section{METODOLOGÍA}

La dieta del pequén fue estudiada por análisis de egagrópilas y restos recolectados en septiembre y noviembre de 2004, en nidos activos en las cuatro islas del área de estudio.

Las egagrópilas fueron humedecidas y posteriormente disgregadas, los restos fueron identificados hasta el mínimo nivel taxonómico posible. Se utilizaron cráneos, hemimandíbulas y dientes, para identificar los mamíferos siguiendo las claves de Reise (1973) y Mann (1978); las aves se identificaron comparando los restos con las colecciones osteológicas del Museo Nacional de Historia Natural (MNHN) y de la Facultad de Ciencias, Universidad de Chile (UCHZV) y utilizando plumas presentes en las egagrópilas, siguiendo el método de Day (1966) y comparando con la colección de plumas (UCHZV). Los artrópodos se identificaron comparando élitros y cabezas con la colección del Instituto de Entomología de la Universidad Metropolitana de Ciencias de la Educación (UMCE).

Para calcular el total de presas presentes en la muestra se consideró el número mínimo de presas posible, en el caso de la aparición de plumas de aves y escamas de reptiles, solo se contabilizaron como un individuo. Los estructuras óseas fueron medidas, utilizando un pie de metro Linz $(0,05$ mm), y se estimó los tamaños corporales de los individuos siguiendo a Mann (1978), Araya \& Millie (1986), Wharman (1990), Yáñez (2000), Mattoni (2002), Pincheira-Donoso \& Núñez (2005), Vidal \& Guerrero (2007), los pesos promedio de las presas (g) se estimaron siguiendo a Schlatter et al. (1982), Muñoz-Pedreros (2000), Muñoz-Pedreros \& Palma (2000) y Gantz \& Martínez (2000) para los mamíferos; Wharman (1990) y Zavalaga (1997) para aves; y a CepedaPizarro et al. (1996) y Carevic et al. (2013), en el caso se los invertebrados (Tabla 2)

\section{Análisis De Datos}

Se calculó el índice numérico (N\%), como el número total de individuos por especie, como un porcentaje del número total de presas consumidas. Biomasa estimada relativa, se obtuvo al multiplicar el número de individuos de cada especie o categoría por su peso estimado (g), dividido posteriormente por la biomasa total y multiplicado por cien (Rau 2000). Se comparó la diversidad dietaria entre islas, 
con el índice $\mathrm{H}^{\prime}$ de Shannon - Weaver (1949), y el número de ítems consumidos (S), adicionalmente se compararon los mismos índices, considerando las diferencias en el tamaño de la muestra (numero de egagrópilas por isla), para esto se dividió el número de individuos de cada ítem por el numero de egagrópilas en cada localidad. Se comparó el índice numérico (porcentaje en la dieta) del ítem yunco (Pelecanoides garnotti) y la densidad de la colonia de esta especie en isla Choros, calculados como individuos por hectárea (Ind ha ${ }^{-1}$ ), como estimador de una probable respuesta funcional del depredador, para esto se contabilizaron los nidos activos de yunco en un radio de $100 \mathrm{~m}$, considerando como centro los nidos de los pequenes, esto cubre una superficie de $31.415 \mathrm{~m}^{2}$ (3,14 ha). Además, se realizó una correlación de Spearman $\left(\mathrm{r}^{2}\right)$ para evaluar la asociación de algunos ítem en la dieta con la densidad de yuncos, utilizando el programa PAST (Hammer et al. 2001).

\section{RESULTADOS}

De las 392 egagrópilas analizadas se obtuvo, que el total de presas consumida correspondió a 3.455 presas, $368(10,7 \%)$ eran vertebrados y $3087(89,3 \%)$ eran invertebrados. El detalle del consumo por taxa en cada isla se presenta en la Tabla 2.

De los restos recolectados en isla Chañaral, se identificaron al menos 20 individuos de la llaca, tres del murciélago vampiro y dos lagartijas de una especie del Género Liolaemus (posiblemente Liolaemus zapallarensis). En isla Choros se identificaron al menos 27 individuos de yunco.

En 32 egagrópilas de las islas Gaviota y Choros se identificó la presencia de carroña (plumas de Spheniscus sp., peces indeterminados, conchas de Gastrópodos, vidrio, plástico y papel), lo cual no fue considerado para el análisis de proporción en la dieta.

\section{Dieta Por IsLa}

El mayor consumo de aves ocurrió en isla Choros (7,16\%), seguido por isla Gaviota ( $3,3 \%$ ) y por isla Damas $2,04 \%$, en isla Chañaral este ítem no apareció en los restos recolectados. Los mamíferos fueron consumidos en mayor proporción en isla Damas. Los insectos fueron el ítem más consumido en todas las islas, la mayor proporción de estos en la dieta se registró en isla Gaviota (80,11\%) (Tabla 2). Isla Choros se registró la mayor diversidad, tanto en número de presas distintas (Choros $\mathrm{S}=20$; Damas $\mathrm{S}=14, \mathrm{p}<0,01,1.000$ iteraciones), como en el índice de diversidad $\left(\mathrm{H}^{\prime} \mathrm{Choros}=\right.$ $2,115)$ siendo significativamente mayor que el de isla Damas $\left(\mathrm{H}^{\prime}=1,743, \mathrm{p}<0,01,1.000\right.$ iteraciones $)$ y que el registrado en isla Gaviota $\left(\mathrm{H}^{\prime}=1,92, \mathrm{p}<0,05,1.000\right.$ iteraciones). Isla
Damas y Gaviota no presentaron diferencias significativas en la diversidad de la dieta.

Al comparar los índices de diversidad $\left(\mathrm{H}^{\prime}\right)$, estandarizados por el tamaño muestral, no se encontraron diferencias significativas en ninguna de las comparaciones entre islas, Choros $\mathrm{H}^{\prime}=2,874$, Damas $\mathrm{H}^{\prime}=2,002$, Gaviota $\mathrm{H}^{\prime}=2,37$ (Choros/Damas $\mathrm{p}=0,07$; Choros/Gaviota $\mathrm{p}=0,448$, Gaviota/ Damas $\mathrm{p}=0,061,1.000$ iteraciones).

\section{Tamaño De Las Presas Y Biomasa}

Las presas más consumidas del total fueron las pequeñas (entre 0 y $50 \mathrm{~mm}$ ), principalmente coleópteros y solífugos con una longitud máxima (LM) estimada de $35 \mathrm{~mm}(\mathrm{n}=2912)$ que aportaron el $16,57 \%$ de la biomasa total. La segunda categoría más consumida de presas, incluye a vertebrados como la llaca y el yunco con una LM entre 197 y $240 \mathrm{~mm}$ $(n=315)$, estas presas corresponde al grupo de mayor aporte energético, representando el $75,15 \%$ de la biomasa total. Los otros rangos de tamaño, entre $51-150 \mathrm{~mm}(\mathrm{n}=216)$ y entre $251-400 \mathrm{~mm}(\mathrm{n}=12)$ solo aportaron el 3,84\% y 4,44\% de la biomasa respectivamente (ver Figura 2).

\section{Ecología Trófica En Isla Choros}

En esta isla se hizo un análisis más detallado, debido a la presencia de un mayor número de parejas de pequén, al mayor número de muestras recolectadas, y a los datos poblacionales de una de las principales presas en esta isla, el yunco.

En total se registraron 15 nidos de pequén (con un total 30 individuos aproximadamente; densidad $=10,3 \mathrm{Ind} / \mathrm{km}^{2}$, 0,103 Ind ha $^{-1}$ ), la totalidad de nidos de pequén se ubican en el sector Suroeste de la isla, y estaban asociados al 95\% ( $\mathrm{n}=$ 4.372) de los nidos activos de yunco registrados (Figura1B).

El yunco es una de las principales presas en esta isla, solo en dos nidos de pequén (NP) la proporción de consumo fue menor al $1 \%$ del total de presas consumidas, estos nidos estaban ubicados en zonas de baja densidad de yuncos (11 y 35 Ind ha-1). En ocho nidos de pequén la proporción de yuncos consumidos fue superior al $8 \%$ del total de presas consumidas por sitio (Ver Tabla 3 y Figura 3)

Se obtuvo una asociación negativa y significativa entre el número de yuncos consumidos y el número de presas/ egagrópila ( $\mathrm{r}^{2}:-0,61, \mathrm{n}=10, \mathrm{p}<0,05,9.999$ iteraciones), también se observó una alta correlación positiva entre los yuncos consumidos y la densidad de estos en el área de estudio ( $r^{2}: 0,67, n=10, p<0,05,9.999$ iteraciones), no existe asociación significativa entre los invertebrados consumidos y la densidad de yuncos en el área de estudio $\left(\mathrm{r}^{2}:-0,41, \mathrm{n}=\right.$ $10, \mathrm{p}<0,21,9.999$ iteraciones). 


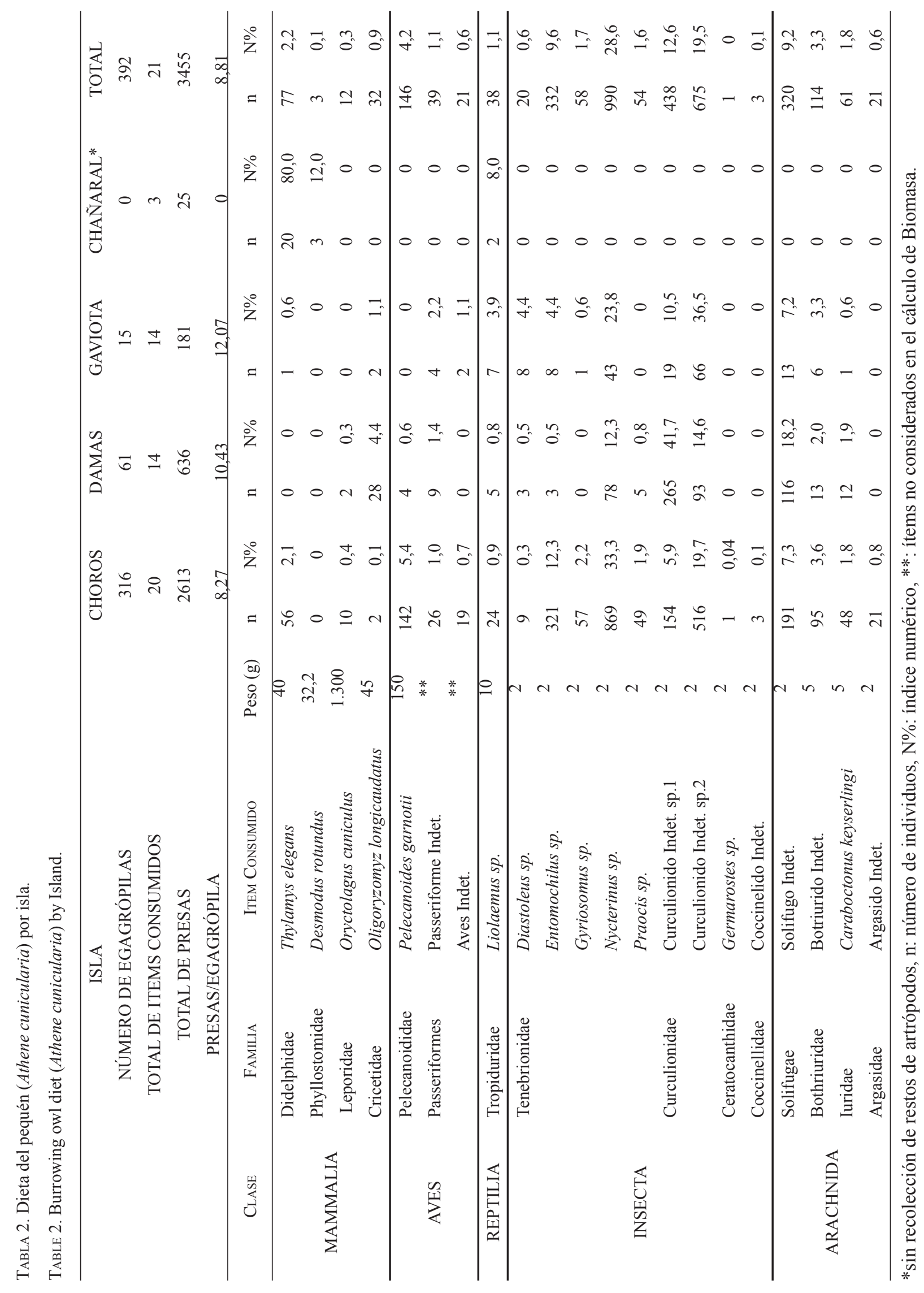




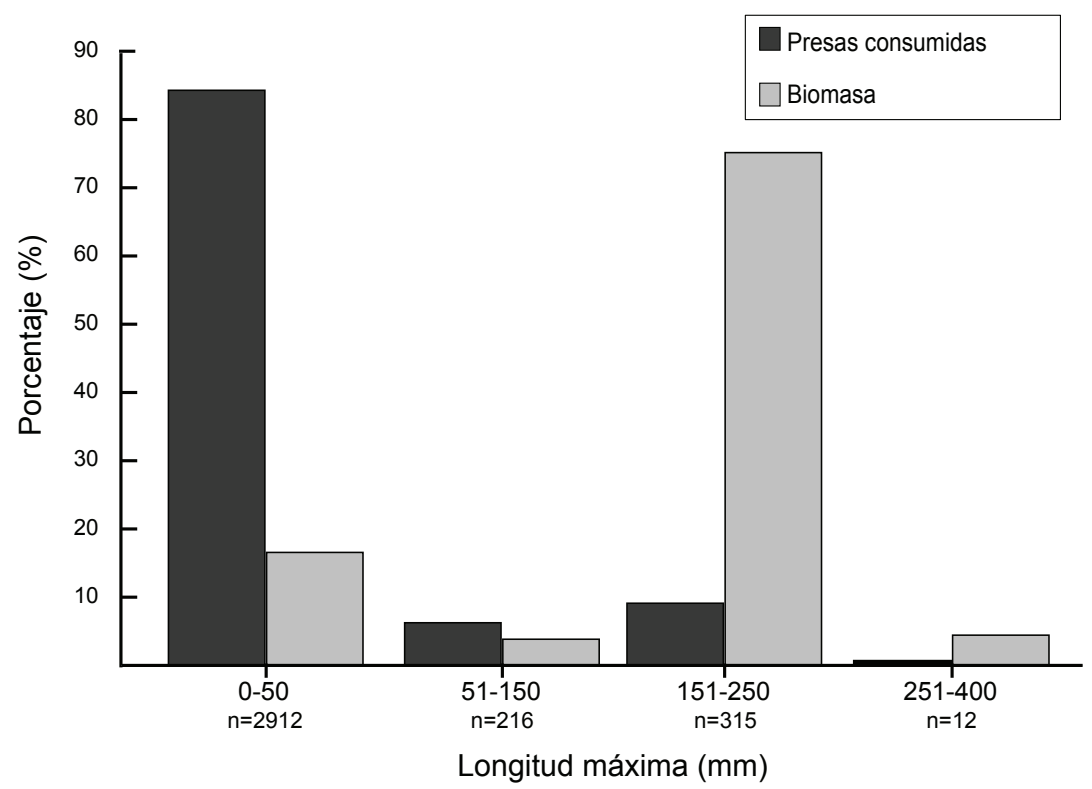

FIgURA 2: Tamaños de las presas y biomasa consumidas por del pequén (Athene cunicularia) en las cuatro islas.

FIGURE 2: Preys size and biomass consumed by the Burrowing owl (Athene cunicularia) in the four island.

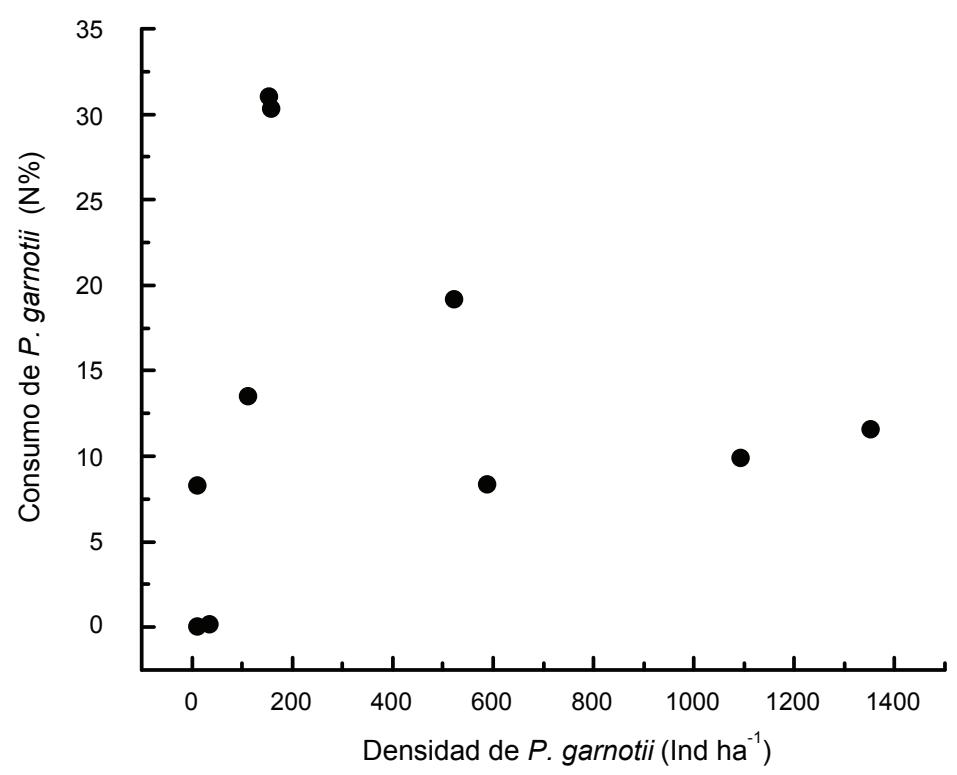

Figura 3: Porcentaje en la dieta y densidades de yunco (Pelecanoides garnotii) en isla Choros.

Figure 3: Percentage in diet and Peruvian diving petrel (Pelecanoides garnotii) densities on Choros Island.

\section{DISCUSIÓN}

Los resultados obtenidos muestran que el pequén consume presas en proporción similar a lo registrado en localidades continentales de Chile (75-95\% de invertebrados y $8-25 \%$ de vertebrados) (Yánez \& Jaksic 1979, Schlatter et al. 1980; Schlatter et al. 1982, Núñez \& Yánez 1982; Jaksic 1986, Torres-Contreras et al. 1994; Silva et al. 1995; Zunino \&
Jofré 1999, Muñoz-Pedrero \& Rau 2004; Rau et al. 2005; Carevic, 2011; Carevic et al. 2013).

Considerando el numero de presas consumidas por tamaño corporal, existe un marcado dominio de presas de pequeño tamaño $(\mathrm{LM}<50 \mathrm{~mm}$ ), principalmente artrópodos (coleópteros, solífugos y argásidos, etc.), sin embargo en términos de biomasa destacan las presas relativamente 
grandes $(\mathrm{LM}=151$ a $250 \mathrm{~mm})$, como el yunco y la llaca, grupo que aportó el $75 \%$ de la biomasa total, constituyendo los ítems más importantes en la dieta del pequén (Figura 2). La presa menos consumida correspondió al conejo $(<251 \mathrm{~mm})$, posiblemente por la dificultad de captura y manipulación de esta especie. En zonas áridas el aporte de los vertebrados va de un 55 a un $75 \%$ de la biomasa total, siendo menor durante los meses de invierno (Carevic et al. 2013).

De la dieta analizada en cada isla, la registrada en isla Choros es la que presentó una mayor diversidad dietaria (índice de diversidad y numero de ítems consumidos); y la menor ocurrió isla Gaviota e isla Chañaral, no obstante en esta última no se recolectaron invertebrados, solo restos de cadáveres asociados al único nido de pequén encontrado en la isla. A pesar de este resultado, al estandarizar por el número de egagrópilas, no se observaron diferencias en la diversidad de presas consumidas entre islas. Las familias de coleópteros Tenebrionidae y Curculionidae, fueron las más consumidas (ver Tabla 2). Destacamos la presencia en las cuatro islas de la llaca, Thylamys elegans que representó el 2,2\% de las presas totales y la presencia de cráneos del murciélago vampiro, Desmodus rotundus, que sólo se registraron en isla Chañaral, lo que reafirma lo propuesto por Luna-Jorquera et al. (2012) respecto de la presencia de esta especie en dicha isla, además adiciona a especies del Orden Chiroptera como presas del pequén, situación no registrada con anterioridad en el norte de Chile. Cabe destacar que en esta isla existe una población de estos murciélagos asociada a la presencia de apostaderos de lobos marinos comunes (Otaria byronia) y de lobos finos australes (Arcthocephalus australis) (Vilina datos sin publicar). Además, en las islas existe un bajo consumo de lagartijas ( $1,1 \%$ del total de presas), a pesar de estas son relativamente abundantes en las cuatro islas.

En el presente estudio se registró Thylamys elegans en la dieta de pequén en isla Choros, esta especie no había sido registrada con anterioridad en esta isla (Luna-Jorquera et al. 2012) por lo que es necesario confirmar este registro indirecto, para establecer su presencia.

En nuestro estudio la proporción de consumo del ratón de cola larga en isla Choros fue menor al $1 \%$, por debajo al 7,17\% establecido por Zunino \& Jofré (1999), esto puede sugerir que esta presa fue reemplazada en la dieta por el yunco, Pelecanoides garnotii, el cual no fue registrado como presa en dicho estudio, y que en el presente trabajo representó el $7,6 \%$ de las presas consumidas totales. El comportamiento de depredación de los pequenes se relaciona con el comportamiento nocturno de los yuncos adultos, quienes vuelven a la isla durante el crepúsculo y la noche (Schiappacasse 2007; Torrés 2010).

Se observa que el ítem aves cobró importancia en la dieta de los pequenes presentes en isla Choros, este ítem representó entre el 8 y el $31 \%$ del total de presas, lo que reafirma su conducta oportunista y sugiere que en condiciones insulares, utilizaría los recursos más abundantes, en este caso aves marinas nidificantes, en estas condiciones el ítem Aves puede tener una participación en la dieta muy superiores al 5\% sugerido por otros autores (Muñoz-Pedreros \& Rau, 2004). El mayor consumo de yunco, se asocia a un menor número promedio de presas por egagrópilas.

La proporción de yuncos en la dieta estaría altamente relacionada con la disponibilidad de esta presa en el ambiente, obteniéndose una curva de consumo similar a la respuesta funcional de tipo III del depredador (FernándezArhex \& Corley 2004). El menor consumo (8\%) se observó donde la densidad de esta presa no supera los $12 \mathrm{Ind} \mathrm{ha}^{-1}$, el consumo máximo (30-31\%) ocurrió donde la densidad de presas fue de $150 \mathrm{Ind} \mathrm{ha}^{-1}$ aproximadamente. La respuesta del pequén de aumentar el consumo frente al aumento de presas, ha sido registrada en zonas continentales con el ítem roedores, donde la relación sería lineal y positiva (Silva et al. 1995; Arana et al. 2006), hay que destacar que en los estudios antes mencionados las densidades de roedores no superaban los 400 Ind ha ${ }^{-1}$. En el presente estudio el consumo de yunco no tiene una relación lineal con las densidades de este en el ambiente, ya que en densidades altas (>500 Ind ha-1), el consumo tiende a caer (desde 30\% máximo) y estabilizarse entre el 8 y el 19\% (ver Fig. 3). Esta respuesta puede estar explicada por la conducta gregaria del yunco, que al agruparse en colonias de alta densidad podría dificultar la capacidad del depredador para atrapar individuos, aumentando el tiempo y la energía de captura, disminuyendo con esto el consumo.

Nuestro estudio asigna gran importancia a las aves marinas en la dieta del pequén en islas, situación ya descrita con especies de las familias Alcidae e Hydrobatidae en el Hemisferio Norte (Mellink 2010), lo que indica que el ítem aves serían utilizado de igual manera que otros vertebrados (roedores) en aéreas continentales, descartando lo sugerido por Pavez \& Gabella (1999), que lo asignan como carroña y reafirmando lo propuesto anteriormente para la relación predador-presa entre el pequén y el yunco en isla Choros (Torrés 2010).

La distribución y abundancia del pequén en este sistema insular no fue homogénea, y sugiere una respuesta numérica frente a la colonia de yuncos de isla Choros. El tamaño de la población de yunco en esta isla se ha estimado en 1.500 parejas reproductivas (Simeone et al. 2003), antes de que las islas fueran protegidas, las colonias de yunco de isla Choros era de menor tamaño (Collar et al. 1992, Vilina, datos sin publicar), y sólo una pareja de pequenes fue registrada (Vilina et al. 1995) a diferencia de lo registrado en el 
TABLa 3. Dieta del pequén (Athene cunicularia) en isla Choros por sitio de muestreo (nido NP).

TABLE 3. Burrowing owl diet (Athene cunicularia) at Choros island by sampling site (nest NP).

\begin{tabular}{|c|c|c|c|c|c|c|c|c|c|c|}
\hline Nido pequén & NP1 & NP2 & NP3 & NP4 & NP5 & NP6 & NP7 & NP8 & NP9 & NP10 \\
\hline $\mathrm{N}^{\mathrm{o}}$ de egagrópilas & 14 & 33 & 37 & 45 & 17 & 40 & 22 & 9 & 60 & 39 \\
\hline $\mathrm{N}^{\mathrm{o}}$ ítems consumidos*. & 7 & 14 & 10 & 15 & 13 & 13 & 11 & 7 & 13 & 14 \\
\hline Total de presas & 33 & 171 & 779 & 136 & 147 & 244 & 180 & 29 & 688 & 206 \\
\hline Presas/egagrópila & 2,36 & 5,18 & 21,05 & 3,02 & 8,65 & 6,1 & 8,18 & 3,22 & 11,47 & 5,28 \\
\hline Invertebrados (N\%) & 60,6 & 82,44 & 97,69 & 73,52 & 83,66 & 84,84 & 87,22 & 68,97 & 91,57 & 84,95 \\
\hline Mamíferos (N\%) & 0 & 1,75 & 0,64 & 0,74 & 0 & 0,82 & 2,22 & 0 & 6,54 & 3,88 \\
\hline Reptiles (N\%) & 3,03 & 1,17 & 0 & 3,68 & 4,08 & 1,64 & 0 & 0 & 0,29 & 1,94 \\
\hline Otras Aves (N\%) & 6,06 & 1,17 & 1,67 & 2,95 & 0,68 & 2,87 & 2,22 & 0 & 1,45 & 0,98 \\
\hline P. garnotii (N\%) & 30,3 & 13,45 & 0 & 19,12 & 11,56 & 9,84 & 8,33 & 31,03 & 0,15 & 8,25 \\
\hline Densidad de $P$. garnotii (Ind ha ${ }^{-1}$ ) & 159,16 & 112,05 & 11,46 & 523,32 & 1352,86 & 1095,02 & 588,25 & 155,34 & 35,65 & 11,46 \\
\hline
\end{tabular}

*Presas diferentes, N\%: índice numérico.

presente estudio donde se observaron al menos 15 parejas de pequén.

La presencia del pequén en las otras islas estudiadas fue menor, a pesar que presentan similitudes en ambientes disponibles, distancia del continente y superficie total (ver Tabla 1) y también en la riqueza de aves y mamíferos terrestres (Luna-Jorquera et al. 2012). La distribución dentro isla Choros tampoco fue homogénea, los nidos de pequén se ubicaron en el sector Suroeste de la isla lugar de concentración del 95\% de los nidos de yunco (ver Fig. 1B). Este patrón podría estar relacionado con la disponibilidad de esta presa, y con la facilitación en la construcción de los nidos, ya que ambas especies construyen nidos subterráneos, situación que se asemeja a la registrada entre el pequén y colonias de mamíferos fosoriales de mediano tamaño (Orth \& Kennedy 2001, Machicote et al. 2004, Poulin et al. 2005).

Con la información recopilada, se mantiene el criterio de considerar al pequén, como un generalista, con comportamiento selectivo sobre presas de alto aporte energético, sugiriendo una respuesta funcional y numérica frente a altas densidades de presas en el ambiente, en nuestro caso frente a una especie de ave marina. Es importante destacar que el yunco está considerado En Peligro a nivel mundial (Birdlife International, 2013; UICN 2013), por lo que se hace necesario profundizar en las interacciones predador-presa, aumentando la información con muestreos en el largo plazo, adicionando información estacional de presas alternativas e incorporando información de otros depredadores, sobre todo en ambientes de área restringida, como lo son las islas.

\section{AGRADECIMIENTOS}

A Mariano Riveros y Esteban Sagredo por apoyar esta empresa. Un especial agradecimiento a Jaime Solervicens, Enrique Silva Aránguiz y Michel Sallaberry, por su ayuda en la identificación de algunos ítems de presas. A Jaime Machuca, Carlos Moscoso y Carolina Zuilt "CDCZ" por su colaboración en el análisis de las muestras, a María José Vargas y Guisela Sinning por su ayuda en la elaboración de los mapas. Este estudio fue financiado por el Ministerio de Bienes Nacionales, en el contexto del subprograma "Viajes Exploratorios 2004".

\section{BIBLIOGRAFÍA}

Arana, M., Ruiz-Luna, M.L., Maria, S.S. \& Ramirez, O. 2006. Population fluctuations of the house mouse in a Peruvian loma and the functional response of burrowing owls. Austral Ecology 31:956-963.

Araya, B. \& Duffy, D.C. 1987. Animal introduction to Isla Chañaral, Chile; their history and effect on seabirds. Cormorant 15: 3-6.

Araya, B. \& Millie, G. 1986. Guía de campo de las aves de Chile. Editorial Universitaria, Santiago, Chile.

Birdlife International. 2013. Species factsheet: Pelecanoides garnotii. Downloaded from http://www.birdlife.org on 23/08/2013.

Capella, J.J., Vilina, Y.A. \& Gibbons, J. 1999. Observación de cetáceos en Isla Chañaral y nuevos registros para el área de la Reserva Nacional Pingüino de Humboldt, norte de Chile. Estudios Oceanológicos 18: 57-64.

CAREvic, F. 2011. Rol del pequén (Athene cunicularia) como controlador biológico mediante el análisis de sus hábitos alimentarios en la Provincia de Iquique, norte de Chile. 
Idesia (Chile), 29: 15-21

Carevic, F., Carmona, E.R. \& Muñoz-Pedreros, A. 2013. Seasonal diet of the burrowing owl Athene cunicularia Molina, 1782 (Strigidae) in a hyperarid ecosystem of the Atacama desert in northern Chile. Journal of Arid Environments 97: 237-241.

Cepeda-Pizarro, J.G., Vasquez, H., Hector Veas, H. \& Colon, G.O. 1996. Relaciones entre tamaño corporal y biomasa en adultos de Tenebrionidae (Coleoptera) de la estepa costera del margen meridional del desierto chileno. Revista Chilena de Historia Natural 69:67-76.

Collar, N.J., Gonzaga, L.P., Krabbe, N., Madroño-Nieto, A., Naranjo, L.G., Parker Iit, T.A. \& Wege, D.C. 1992. Threatened birds of the Americas. The ICBP/IUCN red data book. ICBP, Cambridge, U. K.

DAY, M.F. 1966. Identification of hair and feather fragments in the guts and faeces of stoats and weasels. Journal of Zoology (Lond.) 148: 201-217.

Fernandez-Arhex, V. \& Corley J.C. 2004. La respuesta funcional: una revisión y guía experimental. Ecología Austral, 14(1): 83-93

Gajardo, R. 1994. La Vegetación Natural de Chile. Clasificación y Distribución Geográfica. Editorial Universitaria, Santiago.

Gantz, A. \& Martínez, D.R. 2000. Orden Chiroptera. En: Mamíferos de Chile (Eds. Muñoz-Pedreros A \& J Yañez), pp: 53-65. CEA Ediciones, Valdivia Chile.

Goodall, J., Johnson, A. \& Phillipi, R. 1957. Las aves de Chile, su conocimiento y sus costumbres. Vol. II. Estab. Graf. Platt. Buenos Aires, Argentina.

HAMmer, O., Harper, D. \& RYAN, P. 2001. PAST: paleontological statistics software for education and data analysis. Paleontología Electrónica 4: 1-9.

JAKSIC, F.M. 1986. Predation upon small mammals in shrublands and grasslands of southern South America: ecological correlates and presumable consequences. Revista Chilena de Historia Natural 59: 209-221.

Luna-Jorquera, G., Fernández, C. \& Rivadeneira, M. 2012. Determinants of the diversity of plants, birds and mammals of coastal islands of the Humboldt current systems: implications for conservation. Biodiversity and Conservation 21:13-32.

Machicote, M., Branch, L.C. \& Villarreal, D. 2004. Burrowing owls and burrowing mammals: are ecosystem engineers interchangeable as facilitators?. Oikos, 106: 527-535.

MAnN, G. 1978. Los pequeños mamíferos de Chile (marsupiales, quirópteros, edentados y roedores). Gayana Zoología (Chile) 40: 1-342.

MatToni, C.M. 2002. Bothriurus picunche sp. nov., a New Scorpion from Chile (Bothriuridae). Studies on Neotropical Fauna and Environment, 37(2): 169-174

Mellink, E. 2010. Depredación de aves marinas nocturnas en islotes de isla Guadalupe, México. Revista Mexicana de Biodiversidad, 81(2): 505-509.

Millie, G. 1938. Las aves del Valle del Huasco y sus alrededores (Provincia de Atacama). Rev. Chilena de Historia Natural, 42: 181-205.

Muñoz-Pedreros, A. 2000. Orden Rodentia. En: Mamíferos de Chile (Eds. Muñoz-Pedreros, A. \& Yáñez, J.), pp 73-126. CEA Ediciones, Valdivia Chile.

Muñoz-Pedreros, A. \& Palma, R.E. 2000. Marsupiales. En:
Mamíferos de Chile (Eds. Muñoz-Pedreros, A. \& Yáñez, J.), pp 43-51. CEA Ediciones, Valdivia Chile.

Muñoz-Pedreros, A. \& RaU, J. 2004. Estudio de egagrópilas. En: Aves Rapaces de Chile (Eds. Muñoz-Pedreros A., Rau J. \& J. Yáñez), pp. 263-278. CEA Ediciones. Valdivia, Chile.

NuÑEz, H. \& YaÑEZ, J. 1982. Dieta de Athene cunicularia (Molina 1782) en la V Región (Aves: Strigiformes). Noticiario Mensual del Museo Nacional de Historia Natural (Chile) 26(305): 6-7.

Orth, P.B. \& Kennedy, P.L. 2001. Do land-use patterns influence nest-site selection by burrowing owls (Athene cunicularia hypugaea) in northeastern Colorado? Canadian Journal of Zoology 79: 1038-1045

PavÉz, E. \& Gabella, J.P. 1999. Presencia de aves marinas en la dieta del Pequén (Athene cunicularia) en la costa de la Octava Región. Boletín Chileno de Ornitología 6: 42-43.

Pincheira-Donoso, D. \& NuñEZ, H. 2005. Las especies chilenas del género Liolaemus Wiegmann. 1834 (Iguania: Tropiduridae: Liolaeminae). Taxonomía, sistemática y evolución. Publicación Ocasional, Museo Nacional de Historia Natural (Chile) 59: 1-486.

Poulin, R.G., Todd, L.D., Dohms, K.M., Brigham, R.M. \& Wellicome, T.I. 2005. Factors associated with nestand roost-burrow selection by burrowing owls (Athene cunicularia) on the Canadian prairies. Canadian Journal of Zoology, 83 (10): 1373-1380.

RAu, J. 2000. Métodos de análisis en ecología trófica. En: Mamíferos de Chile (Eds. Muñoz-Pedreros, A. \& Yáñez, J.), pp 397-406. CEA Ediciones, Valdivia Chile.

Reise, D. (1973) Clave para la determinación de los cráneos de marsupiales y roedores chilenos. Gayana Zoología (Chile) 27: $1-20$

Rau, J., Muñoz-Pedreros, A. \& Martínez, D.R. 2005. Diversidad trófica de aves rapaces y mamíferos carnívoros en la Cordillera de la Costa. Recuadro 9, pp: 537-538, en: Smith-Ramírez, C., Armesto, J.J. \& Valdovinos, C. (eds.). Historia, Biodiversidad y Ecología de los Bosques Costeros de Chile. Editorial Universitaria. Santiago, Chile. $708 \mathrm{pp}$.

Schiappacasse, D. 2007. Patrones de actividad diarios y uso de hábitat marino por parte del petrel buceador Pelecanoides garnotii (Lesson 1896) en el sistema costero de Coquimbo. Tesis. Universidad Católica del Norte. Coquimbo, Chile.

Schlatter, R., Yañez, J., Nuñez, H. \& JaKsic, F.M. 1979. The diet of the Burrowing Owl in central Chile and its relation to prey size. Auk: 97: 616-619.

Schlatter, R., NuÑez, H. \& JAKsic, F.M. 1982 Estudio estacional de la dieta del Pequén, Athene cunicularia (Molina) (Aves, Strigidae) en la Precordillera de Santiago. Medio Ambiente (Chile), 6 (1): 9-18.

Silva, S.I., Lazo, I., Silva-Aranguiz, E., Jaksic, F.M., Meserve, P.L. \& Gutierrez, J.L. 1995. Numerical and functional response of burrowing owls to long-term ma mmal fluctuations in Chile. Journal of Raptor Research 29: 250255.

Simeone, A,. Luna-Jorquera, G., Bernal, M., Garthe, S., Sepúlveda, F., Villablanca, R., Ellenberg, U., Contreras, M., Muñoz J. \& Ponce, T. (2003) Breeding distribution and abundance of seabirds on islands off north-central Chile. Revista Chilena de Historia Natural, 76: 323-333. 
TorRés MJ. 2010. Dieta y conducta de predación de Athene cunicularia sobre Pelecanoides garnotii en isla Choros, norte de Chile. Tesis. Universidad Católica del Norte. Coquimbo, Chile.

Torres-Contreras, H., Silva-Aranguiz, E. \& Jaksic, F.M. 1994. Dieta y selectividad de presas de Speotyto cunicularia en una localidad semi-árida del norte de Chile a lo largo de siete años (1987-1993). Revista Chilena de Historia Natural 67: 329-340.

UICN (2013) IUCN Red List of Threatened Species. Version 2013.1. <www.iucnredlist.org>. Downloaded on 23 August 2013.

Vidal, P. \& Guerrero, M. 2007. Los Tenebriónidos de Chile, Ediciones Universidad Católica de Chile, Santiago, 478p.

VILINA, Y.A. 1992. Status of the Peruvian diving petrel Pelecanoides garnotii, on Chañaral Island, Chile. Colonial Waterbirds 15: 137-139.

Vilina, Y.A., Capella, J.J., GonzÁlez, J. \& GibBons, J. 1995.
Apuntes para la conservación de las aves de la Reserva Nacional Pingüino de Humboldt. Boletín Chileno de Ornitología 2: 2-6.

Warham, J. 1990. The Petrels, their ecology and breeding systems. Academic Press. London. 440pp

Yañez, J., Tamayo, M. \& NuÑez, H. 2000. Clave de determinación de los mamíferos chilenos. Pp 325-346, en: Mamíferos de Chile. Muñoz-Pedreros A \& J Yañez. Ediciones CEA, Valdivia Chile. $464 \mathrm{pp}$

YAÑEZ, J. \& JAKSIC, F.M. 1979. Predación oportunista de Speotyto cunicularia en el norte chico (Aves: Strigiformes). Noticiario Mensual del Museo Nacional de Historia Natural (Chile) 272: 12.

Zavalaga G.B. 1997. Maximum dive depths of the Peruvian diving-petrel. The Condor 99:1002-1004

Zunino, S. \& Jofré, C. 1999 Dieta de Athene cunicularia en isla Choros, Reserva Nacional Pingüino de Humboldt, IV Región. Boletín Chileno de Ornitología 6: 2-7.

Recibido: 06.12.13

Aceptado: 15.05 .14 